\title{
COVID-19 in Pregnant Women: Case Series from One Large New York City Obstetrical Practice
}

\author{
Nathan S. Fox, $\mathrm{MD}^{1,2}$ Stephanie Melka, $\mathrm{MD}^{1,2}$ \\ ${ }^{1}$ Maternal Fetal Medicine Associates, PLLC, Gynecology, and Reproductive \\ Science, Icahn School of Medicine at Mount Sinai, New York \\ 2 Department of Obstetrics, Gynecology, and Reproductive Science, \\ Icahn School of Medicine at Mount Sinai, New York \\ Address for correspondence Nathan S. Fox, MD, Maternal Fetal \\ Medicine Associates, PLLC, 70 East 90th Street, New York, NY 10128 \\ (e-mail: nfox@mfmnyc.com). \\ Am J Perinatol 2020;37:1002-1004.

\begin{abstract}
Keywords

- COVID-19

- coronavirus

- pregnancy

- infection

Objective This study aimed to report a case series of pregnant women in New York City with confirmed or presumed coronavirus disease (COVID-19) infection.

Study Design Beginning March 22, 2020, all pregnant women from one large obstetrical practice in New York City were contacted regularly to inquire about symptoms of COVID-19 (fever, cough, shortness of breath, malaise, anosmia), or sick contacts. A running log was kept of these patients, as well as all patients who underwent COVID-19 testing. For this report, we included every patient with suspected COVID-19 infection, which was defined as at least two symptoms, or a positive COVID19 nasopharyngeal polymerase chain reaction test.

Results From March 22, 2020 until April 30, 2020, 757 pregnant women in our practice were evaluated and 92 had known or suspected COVID-19 (12.2\%, 95\% confidence interval [CI]: 10.0-14.7\%). Of these 92 women, 33 (36\%) had positive COVID-19 test results. Only one woman required hospital admission for 5 days due to COVID-19 $(1.1 \%, 95 \% \mathrm{Cl}$ : $0.2-5.9 \%)$. One other woman received home oxygen. No women required mechanical ventilation and there were no maternal deaths. One woman had an unexplained fetal demise at 14 weeks' gestation around the time of her COVID-19 symptoms. Twenty one of the 92 women have delivered, and all were uncomplicated.

Conclusions Among 92 women with confirmed or presumed COVID-19, the overall morbidity was low. These preliminary results are encouraging for pregnant women
\end{abstract} \\ during the COVID-19 pandemic.
}

\section{Key Points}

- Coronavirus disease (COVID-19) is prevalent in New York City.

- In this case series, COVID-19 in pregnant women had a very low morbidity and no mortality.

- This preliminary data is reassuring for pregnant women at risk of COVID-19.

In the past several weeks, COVID-19 has spread throughout New York City. One recent report indicated that $13.7 \%$ of women presenting to Labor and Delivery in a New York Hospital tested positive for COVID-19. ${ }^{1}$ However, the risks associated with COVID-19 in pregnancy are currently unknown. Initial data are likely to be case reports, followed by case series. ${ }^{2}$ However, case reports can sometimes lead to an overestimation of risk as uncomplicated cases are less likely to received

May 6, 2020

accepted after revision

May 6, 2020

published online

May 21, 2020
Copyright $\odot 2020$ by Thieme Medical

Publishers, Inc., 333 Seventh Avenue, New York, NY 10001, USA. Tel: +1(212) 760-0888.
DOI https://doi.org/ 10.1055/s-0040-1712529. ISSN 0735-1631. 
be reported. Furthermore, series derived from hospital admissions could overestimate risk, as these tend to be the sickest patients. The objective of this Research Letter is to report our experience with COVID-19 in a large New York City obstetrical practice with $\sim 1,000$ deliveries per year.

\section{Materials and Methods}

Beginning on March 22, 2020 we began questioning every pregnant patient in our practice for symptoms suggestive of COVID-19 (fever, cough, shortness of breath, malaise, anosmia), or sick contacts. We did this in three ways: (1) We sent an email to all patients asking them to contact us if they had any of these symptoms; (2) We called every patient by telephone in advance of all scheduled prenatal visits; (3) We asked every patient at every office visit. New York City Department of Health guidelines were followed in regard to isolation and quarantine for women with presumed COVID-19 or sick contacts. For all patients with suspected COVID-19 or sick contacts, we contacted them every 2 to 3 days and kept a running list of these patients including symptoms, treatments, and test results, if performed. After Biomedical Research Alliance of New York Institutional Review Board approval was obtained, we reviewed the charts for these patients and report the results here. For this report, we included every patient with suspected COVID-19 infection, which was defined as at least two of the following symptoms (fever, cough, shortness of breath, malaise, anosmia), or a positive COVID-19 nasopharyngeal polymerase chain reaction (PCR) test. We did not include women who only had sick contacts.

\section{Results}

From March 22, 2020 until April 30, 2020, 757 pregnant women in our practice were evaluated for possible COVID-19 infection either with an in-person visit or virtual visit over a video platform. Of these 757 women, 92 had known or suspected COVID-19, yielding an incidence of $12.2 \%$ (95\% confidence interval [CI]: 10.0-14.7\%). Of these 92 women, 33 (36\%) had positive COVID-19 test results and 51 women (55\%) were not tested, as testing was not routinely available in the community. An additional 8 (9\%) women tested negative, but had classic symptoms of COVID-19, as well as household members with positive COVID-19 PCR test results. These eight women were considered to likely have false negative tests. All other women with negative test results were considered true negatives and not included. Of the 33 women who tested positive, 27 (82\%) were tested due to symptoms of COVID-19, and 6 (18\%) were asymptomatic, but were found to be PCR positive at the time of admission for delivery. Characteristics of the 92 women with suspected COVID-19 are shown in -Table 1.

Twenty one of the 92 women have delivered and all were uncomplicated. Neonatal testing is not available at this time. Only one woman of the 92 women required hospital admission due to COVID-19 (1.1\%, 95\% CI: 0.2-5.9\%). She is a 40 -year-old with obesity, type I diabetes, and hypothyroidism. She was admitted at 29 weeks' gestation for 5 days due to COVID-19 and respiratory difficulty. She received oxygen by nasal cannula, azithromycin, and hydroxychloroquine, and was discharged 5 days later. She did not require mechanical ventilation. One other woman received home oxygen therapy by nasal cannula. One woman had an unexplained fetal demise at 14 weeks' gestation around the time of her COVID-19 symptoms. There were no maternal deaths.

\section{Discussion}

We report on 92 pregnant women with known or suspected COVID-19. Our incidence of $12.2 \%$ is in line with other reports from New York City. ${ }^{1}$ However, we suspect the incidence of COVID-19 is actually higher than $12.2 \%$ in

Table 1 Characteristics of the population

\begin{tabular}{|llll|}
\hline & $\begin{array}{l}\text { Total population of } \\
\text { COVID-19 pregnant women } \\
\boldsymbol{n}=92\end{array}$ & $\begin{array}{l}\text { Positive PCR test for COVID-19 } \\
\boldsymbol{n}=33\end{array}$ & $\begin{array}{l}\text { Suspected COVID-19 } \\
\boldsymbol{n}=59\end{array}$ \\
Mean age (years) & $31.4(6.5)$ & $31.0(6.2)$ & $31.7(6.7)$ \\
Symptoms of COVID-19 & $86(93.5)$ & $27(81.8)$ & $59(100)$ \\
Fever & $40(43.5)$ & $13(39.4)$ & $27(45.8)$ \\
Cough & $79(85.9)$ & $27(81.8)$ & $52(88.1)$ \\
Shortness of breath & $7(7.6)$ & $1(3.0)$ & $6(10.2)$ \\
Malaise & $74(80.4)$ & $24(72.7)$ & $50(84.7)$ \\
Anosmia & $27(29.3)$ & $8(24.2)$ & $19(32.2)$ \\
Oxygen therapy & $2(2.2)$ & $1(3.0)$ & $1(1.7)$ \\
Admission to hospital & $1(1.1)$ & $1(3.0)$ & $0(0)$ \\
Mechanical ventilation & $0(0)$ & $0(0)$ & $0(0)$ \\
Death & $0(0)$ & $0(0)$ & $0(0)$ \\
Delivered & $21(27.3)$ & $18(54.5)$ & $3(5.1)$ \\
\hline
\end{tabular}

Abbreviations: COVID-19, coronavirus disease; PCR, polymerase chain reaction; SD, standard deviation.

Note: Numbers are mean (SD) or $n(\%)$. 
our population as we did not do routine testing on all patients (aside from those admitted for delivery). To illustrate, we had six women in just a few weeks who tested positive at delivery who had no symptoms. Our cohort had no mortality and an overall low morbidity from COVID-19. No women required mechanical ventilation. Only one woman was admitted to the hospital for 5 days. There was one 14-week fetal demise and it is unclear if this was due to COVID-19 or not. These preliminary results are encouraging for pregnant women during the COVID-19 pandemic. These results differ from other series ${ }^{3}$ that may be due to only the sickest patients begin tested in prior series. With further data collection and larger case series, more precise estimates of incidence, morbidity, and mortality can be obtained, as well as specific risk factors in pregnant women and potential therapies.
Conflict of Interest

None declared.

\section{References}

1 Sutton D, Fuchs K, D'Alton M, Goffman D. Universal screening for SARS-CoV-2 in women admitted for delivery. N Engl J Med 2020. Doi: 10.1056/NEJMc2009316

2 Breslin N, Baptiste C, Gyamfi-Bannerman C, et al. COVID-19 infection among asymptomatic and symptomatic pregnant women: two weeks of confirmed presentations to an affiliated pair of New York City hospitals. Am J Obstet Gynecol MFM 2020;100118:100118; [Epub ahead of print]. Doi: 10.1016/j.ajogmf.2020.100118

3 Di Mascio D, Khalil A, Saccone G, et al. Outcome of coronavirus spectrum infections (SARS, MERS, COVID 1 -19) during pregnancy: a systematic review and meta-analysis. Am J Obstet Gynecol MFM 2020;100107:100107; [Epub ahead of print]. Doi: 10.1016/ j.ajogmf.2020.100107 